la revue La revue pour l'histoire du CNRS

POUR LHISTORE DU CNRS $\quad 24 \mid 2009$

Soixante-dixième anniversaire du CNRS

\title{
Aux quatre coins du monde
}

Anne d'Albis

\section{OpenEdition}

Journals

Édition électronique

URL : https://journals.openedition.org/histoire-cnrs/9123

DOI : 10.4000/histoire-cnrs.9123

ISSN : 1955-2408

Éditeur

CNRS Éditions

Édition imprimée

Date de publication : 5 octobre 2009

ISSN : 1298-9800

Référence électronique

Anne d'Albis, «Aux quatre coins du monde », La revue pour l'histoire du CNRS [En ligne], 24 | 2009, mis en ligne le 05 octobre 2009, consulté le 20 mai 2021. URL : http://journals.openedition.org/histoirecnrs/9123; DOl : https://doi.org/10.4000/histoire-cnrs.9123

Ce document a été généré automatiquement le 20 mai 2021.

Comité pour l'histoire du CNRS 


\title{
Aux quatre coins du monde
}

\author{
Anne d'Albis
}

La revue pour l'histoire du CNRS. Vous êtes biochimiste de formation.

2 Anne d'Albis. En effet, mon travail de thèse a porté sur une étude physico-chimique des relations structure/fonction d'une enzyme pancréatique. J'ai pu établir le maintien d'une conformation active de l'enzyme lors de son interaction avec son substrat, ce qui m'a valu d'être invitée quelques mois à l'université de Cornell (USA). Au cours de mon séjour postdoctoral de trois ans à Londres dans l'unité de biophysique du King's College dirigée par Maurice Wilkins ${ }^{1}$, je me suis ensuite intéressée à la myosine, protéine impliquée dans la contraction musculaire et la motilité cellulaire. J'en ai fait le principal axe de recherche de mon équipe à Orsay de 1976 à 1995. Nous y avons mis au point une technique électrophorétique particulièrement délicate de séparation des isoformes de la myosine. Ce résultat de 1979, présenté à une Gordon Conference, a permis à de nombreux auteurs d'utiliser la myosine comme marqueur de multiples événements physiologiques et pathologiques et d'étudier ainsi aisément la plasticité musculaire.

3 La revue... 32 ans de recherche, puis 10 ans d'administration de la recherche. Comment passe-t-on de l'« autre côté » de la barrière ?

4 A. d'A. Je voudrais souligner que ces deux activités sont liées et qu'on ne peut effectuer la seconde sans une solide expérience de la première; pour faciliter le travail d'un chercheur dans sa recherche, il faut en être un soi-même.

5 La revue... Sur vos 42 années au CNRS, vous en avez passé environ 8 à l'étranger. L'international fait-il partie intégrante de votre histoire personnelle?

6 A. d'A. L'international fait bien évidemment partie intégrante de mon histoire personnelle. J'appartiens en effet à une famille de diplomates et en ai même épousé un... Loin de représenter un inconvénient, la diplomatie m'a habituée enfant à vivre à l'étranger, m'a permis de poursuivre ma formation de chercheur en Grande-Bretagne, et enfin d'être de 1996 à 2001, le premier représentant du CNRS en Afrique du Sud. Ce poste a permis d'initier une coopération de toute première qualité sur des thématiques diverses, comme la biodiversité, la géologie, les maladies infectieuses, la paléontologie, 
la "bonne gouvernance ", etc. L'Afrique du Sud, géant du continent africain, est en effet un « laboratoire vivant ", qui doit également permettre à la France une meilleure coopération avec l'Afrique francophone. Ce poste, je ne l'avais pas choisi, mais je l'ai voulu. Il est tombé à pic après la fin de l'apartheid alors que tout était à construire. Dès la nomination de mon mari en Afrique du Sud, j'ai été contactée par Daniel Pardo, le directeur des relations internationales de l'époque, qui me proposait d'y représenter le CNRS. Passionnée par cette proposition, je l'ai aussitôt acceptée... mais le directeur des sciences de la vie, dont je dépendais, s'y est totalement opposé et j'ai été mise en disponibilité, sans même pouvoir lui en parler. Pour la première et heureusement dernière fois de ma vie professionnelle, j'étais mise à l'écart et j'enrageais de ne pouvoir continuer à travailler au CNRS et pour le CNRS. Je me suis alors mise au travail pendant ces mois de disponibilité et mon rapport sur l'état et l'organisation de la recherche en Afrique du Sud a entraîné un revirement de position de l'administration du CNRS et ma nomination.

7 La revue... États-Unis, Grande-Bretagne, Afrique du Sud... quel bilan dressez-vous de vos expériences ? Y avez-vous connu des difficultés majeures ? Quels en ont été les points forts?

8 A. d'A. Plus de 8 années à l'étranger sur un total de 42 années de vie professionnelle au CNRS me paraît une bonne proportion. En tant que jeune chercheur, passer quelques mois/années dans un laboratoire à l'étranger ne pose aucune difficulté puisque le CNRS vous assure votre poste et donc votre salaire, énorme avantage qu'on nous envie de toutes parts. On y apprend à penser autrement, à raisonner autrement, à collaborer autrement. Ensuite, il faut revenir en France pour y rapporter ses nouvelles expertises et poursuivre les liens engagés. Plus tard, bien plus tard, assumer une fonction administrative à l'étranger permet également de mieux connaître et comprendre ce qui se fait ailleurs et ne pose, en principe, aucune difficulté...

9 La revue... Pour revenir à votre mission en Afrique du Sud, jugez-vous importante l'existence de bureaux du CNRS à l'étranger pour l'image du CNRS ? Pour celle de la France ? Pour la coopération scientifique internationale?

A. d'A. L'existence de bureaux du CNRS à l'étranger est très importante, notamment, mais pas seulement, dans les pays lointains. Lorsque je suis arrivée en Afrique du Sud, j'ai eu la grande surprise de constater que le CNRS n'y était que peu ou même pas du tout connu. J'ai donc eu à coeur de le faire connaître en visitant les laboratoires, d'abord à titre personnel par des séminaires de recherche, puis au titre de représentante du CNRS par des exposés sur son organisation, son budget, son personnel, sa stratégie scientifique, ses programmes de recherche... Ensuite, des visites de chercheurs dans toutes les disciplines, ce qui témoigne du rayonnement du CNRS, des colloques réunissant une dizaine de chercheurs de chaque pays, des missions au plus haut niveau, comme celles des deux directrices générales, Catherine Bréchignac et Geneviève Berger, ont été organisés. Des accords de coopération scientifique ont été signés, des appels à propositions pour des collaborations ont été lancés. Je suis convaincue que la présence sur place dans tel ou tel pays d'un chercheur du CNRS est nécessaire pour démarrer et ensuite amplifier la coopération. Et cela ne coûte qu'un salaire au CNRS! L'image du CNRS en sort évidemment grandie, mais, je dirais, également et un peu pompeusement, celle de la France, puisque, bien évidemment, le bureau du CNRS à l'étranger travaille main dans la main avec le service de coopération 
et d'action culturelle de l'Ambassade de France, et notamment avec l'attaché scientifique, quand il y en a un.

11 La revue... Quels bénéfices en avez-vous tirés à titre personnel ?

12 A. d'A. Un chercheur dans son laboratoire est, la plupart du temps, et c'était mon cas, très spécialisé dans un seul domaine scientifique. Le poste de représentant à l'étranger permet une ouverture considérable sur les autres domaines puisqu'il doit avoir une vision d'ensemble de la recherche du pays où il est accrédité, organiser les missions des visiteurs du CNRS et celles des chercheurs étrangers, participer aux colloques bilatéraux et en faire état. J'ai donc vivement apprécié cette possibilité qui m'était offerte de m'initier à d'autres spécialités que la mienne, de rencontrer des chercheurs de ces spécialités, de mieux comprendre aussi les atouts et les défis rencontrés par le CNRS.

13 La revue... Vous avez terminé votre "vie au CNRS" en tant que directrice adjointe des relations internationales (DRI) à Paris. L'action y est-elle semblable ou différente de celle menée à l'étranger ?

A. d'A. De l'automne 2001 à l'été 2005, j'ai été directrice adjointe de la DRI, en charge de l'Europe. La direction adjointe de la DRI et la responsabilité de l'Europe étaient localisées depuis plusieurs années et jusqu'au printemps 2001 à Bruxelles; les deux responsables des secteurs Europe de l'Ouest et Europe de l'Est, localisés à la DRI à Paris, avaient quitté leur poste à l'été 2000. J'ai donc été amenée à réorganiser le secteur, puis j'ai remis à jour et modernisé tous les accords bilatéraux en y incorporant notamment un chapitre sur la propriété intellectuelle, travaillé avec les départements scientifiques à la mise en place de nouvelles actions de collaboration, entretenu avec nos partenaires européens des relations fortes et fructueuses. Cette période de ma vie " administrative » a été aussi passionnante que la précédente en Afrique du Sud, mais très différente. Beaucoup moins de travail "sur le terrain ", c'est-à-dire auprès des chercheurs dans leurs laboratoires, beaucoup moins de "science ", beaucoup plus de réunions et de gestion. Dans le premier cas, je n'étais chargée que de l'Afrique du Sud principalement, car les autres pays d'Afrique australe s'étaient progressivement ajoutés à mon portefeuille - alors que, à Paris, j'ai été en charge de toute l'Europe, de l'Atlantique à l'Oural, ainsi que des pays d'Asie centrale !

15 La revue... Des modifications intervenues en 2007 ont conduit à une scission entre les relations internationales et les affaires européennes. Quel est votre sentiment?

16 A. d'A. Tout dépend de ce que l'on appelle les « affaires européennes ». En général, on entend par cette expression les relations communautaires. Celles-ci sont gérées à Paris, mais également et principalement par le bureau du CNRS à Bruxelles. En ce qui concerne les autres relations européennes, les relations bilatérales, elles sont gérées à Paris et utilisent les mêmes outils et les mêmes procédures que les relations avec le reste du monde. Par ailleurs, les relations communautaires ne sont pas le seul apanage des pays de l'Union européenne, puisqu'elles concernent aussi et de plus en plus les autres pays du continent européen et les pays des autres continents. Il ne me semble donc pas approprié de séparer en deux directions, les relations internationales et européennes.

La revue... Un dernier mot? 
18 A. d'A. J'ai été et reste passionnément attachée au CNRS. L'organisme présente certainement des imperfections - que je ne vois pas d'ailleurs ou que je ne veux pas voir - mais il a l'immense qualité d'exister. Les dernières années, lorsque je partais en mission en Europe, ou même à la fin de ma mission en Afrique du Sud, et que je me présentais comme appartenant au CNRS, toutes les portes s'ouvraient. Je ne peux donc que souhaiter une longue, très longue vie au CNRS. Springbok en Afrique du Sud. (C) Anne Corval, directrice du bureau du CNRS en Afrique sub-saharienne

De petits coquillages percés, « Nassarius kraussianus ", datés de 75000 ans, ont été découverts dans la grotte de Blombos en Afrique du Sud. Ils étaient utilisés comme objets de parure. Ce sont les plus vieux bijoux jamais découverts. (C) CNRS Photothèque/ Francesco d'Errico, Marian Vanhaeren

\section{NOTES}

1. Prix Nobel de médecine en 1962 avec Crick et Watson.

\section{RÉSUMÉS}

Anne d'Albis a été directrice de recherche au CNRS, représentante du CNRS en Afrique du Sud, et directrice adjointe de la Direction des relations internationales. À la retraite depuis l'été 2005, elle nous livre un regard à la fois attendri, passionné et acéré, sur son parcours professionnel et sur l'institution qu'elle a représentée au-delà des frontières.

\section{AUTEUR}

\section{ANNE D'ALBIS}

Anne d'Albis a été directrice de recherche au CNRS, représentante du CNRS en Afrique du Sud, et directrice adjointe de la Direction des relations internationales. 